02

\title{
Влияние $\beta$-дикетонатного комплекса европия(III) на ориентационные отклики в сигнале сверхбыстрого оптического эффекта Керра*
}

\author{
(С) В.Г. Никифоров ${ }^{1}$, Д.К. Жарков ${ }^{1}$, Д.В. Лапаев ${ }^{1}$, А.Г. Шмелев ${ }^{1}$, А.В. Леонтьев ${ }^{1}$, \\ B.C. Лобков ${ }^{1}$, А.А. Князев ${ }^{2}$, Ю.Г. Галяметдинов ${ }^{1,2}$
}

${ }^{1}$ Казанский фризико-технический институт им. Е.К. Завойского

ФИЦ Казанский научный центр РАН,

420029 Казань, Россия

${ }^{2}$ ФГБОУ ВО „КНИТУ“,

Казанский национальный исследовательский технологический университет, 420015 Казань, Россия

e-mail: vgnik@mail.ru

Поступила в редакцию 24.09.2018 г.

Эксперименты по регистрации сверхбыстрого оптического эффекта Керра (ОЭК) в растворе $\beta$-дикетонатного комплекса европия(III) в толуоле с концентрацией $10^{-2} \mathrm{~mol} / \mathrm{l}$ показали, что совокупный сигнал ОЭК не является суперпозицией двух вкладов, один из которых связан с колебательно-вращательной динамикой молекул толуола, а другой - комплексов европия(III). Это прямо указывает, что комплексы европия(III) существенно меняют структуру жидкости толуола, что отражается на характере релаксации ориентационного отклика в пикосекундном диапазоне.

DOI: 10.21883/OS.2019.01.47046.280-18

\section{Введение}

В настоящее время $\beta$-дикетонатные комплексы лантаноидов(III) находятся в фокусе внимания многочисленных исследовательских групп, поскольку представляют интерес как с фундаментальной, так и с прикладной точек зрения $[1,2]$. В первую очередь это связано с уникальной способностью данных соединений эффективно трансформировать УФ свет, поглощаемый хромофорными группами координированных $\beta$-дикетонатных лигандов, в монохроматическую люминесценцию ионов лантаноидов(III) [1].

В работах [3-9] нами показано, что фотофизические свойства (в том числе эффективность внутримолекулярного переноса энергии от лигандов к иону лантаноида) застеклованных пленок $\beta$-дикетонатных комплексов европия(III) и тербия(III) существенно зависят от геометрии комплексов в образцах. Это позволяет использовать лазерную модификацию тонких застеклованных пленок $\beta$-дикетонатного комплекса тербия(III) для управления яркостью люминесценции путем изменения их локальной структуры [5-7].

Хорошо известно, что растворители также оказывают существенное влияние на фотофизические свойства комплексов лантаноидов(III). Например, в работе [8] показано различие между спектрами поглощения $\beta$ дикетонатного комплекса европия(III) в застеклованной пленке и растворе. Кроме того, известны процессы агре-

* XIII International Conference on Hole Burning, Single Molecule, and Related Spectroscopies: Science and Applications (HBSM-2018), August 6-12, 2018, Suzdal-Moscow, Russia. гации комплексов в растворах, которые также оказывают существенное влияние на их люминесцентные свойства (например, препятствуют внутримолекулярным вращениям и таким образом уменьшают скорость безызлучательной релаксации) [10]. Вышеперечисленные факты ясно свидетельствуют о сильном межмолекулярном взаимодействии комплексов лантаноидов(III) с молекулами растворителя. Естественно ожидать, что данное взаимодействие сказывается на локальной структуре самого растворителя и вращательной динамике молекул.

Для выяснения этого вопроса мы использовали фемтосекундную поляризационную спектроскопию с регистраций сверхбыстрого оптического эффекта Керра (ОЭК) [11-15]. В ней используется оптическая схема „накачивающий импульс-пробный импульс“ для регистрации низкочастотных $\left(0-600 \mathrm{~cm}^{-1}\right)$ оптических откликов. Время-разрешенный сигнал ОЭК формируется нелинейными оптическими откликами среды третьего порядка и отражает степень наведенной лазерным импульсом нестационарной анизотропии среды, которая возникает в результате когерентных колебательных и вращательных движений молекул в жидкости. Для сравнительного анализа мы выбрали чистый толуол и раствор $\beta$-дикетонатного комплекса $\mathrm{Eu}\left(\mathrm{CPDk}_{3-\mathrm{Ph}}\right)_{3} \mathrm{bpy}_{1-1}$ $\left[\mathrm{CPDk}_{3-\mathrm{Ph}}\right.$ - 1-(4-(4-пропилциклогексил)фенил)октан1,3-дионато, bру $1-1$ - 5,5'-диметил-2,2'-бипиридин] [16] (рис. 1) в толуоле с концентрацией $10^{-2} \mathrm{~mol} / \mathrm{l}$. Следует отметить, что низкая концентрация комплексов $\mathrm{Eu}\left(\mathrm{CPDk}_{3-\mathrm{Ph}}\right)_{3} \mathrm{bpy}_{1-1}$ не позволяет получить от них сигнал ОЭК на фоне сильного сигнала ОЭК, обусловленного молекулами растворителя. Это обстоятельство позво- 


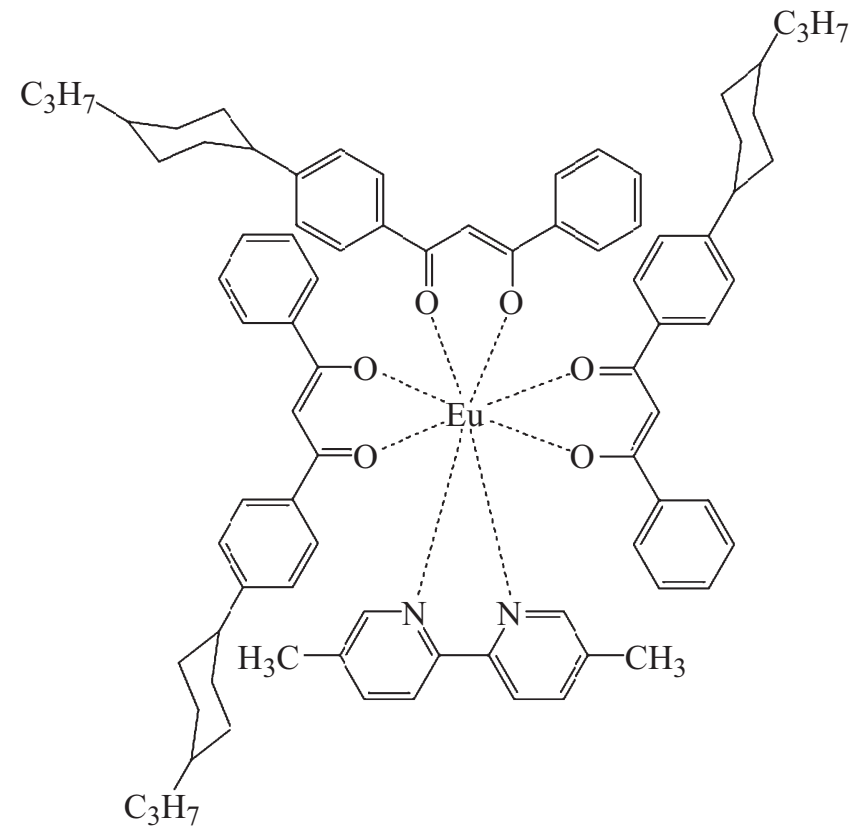

Рис. 1. Структурная формула комплекса $\mathrm{Eu}\left(\mathrm{CPDk}_{3-\mathrm{Ph}}\right)_{3} \mathrm{bpy}_{1-1}$.

лило нам наблюдать существенные изменения в ориентационной динамике молекул толуола в чистой жидкости толуола и растворе комплекса $\mathrm{Eu}\left(\mathrm{CPDk}_{3-\mathrm{Ph}}\right)_{3} \mathrm{bpy}_{1-1}$ в толуоле.

\section{Экспериментальная часть}

В качестве источника фемтосекундных импульсов использовался титан-сапфировый лазер (производство Авеста-Проект, Троицк, Россия). Средняя энергия лазерных импульсов длительностью $45 \mathrm{fs}$ на полувысоте с длиной несущей волны $790 \mathrm{~nm}$ составила $4 \cdot 10^{-9} \mathrm{~J}$. В основе эксперимента лежала традиционная оптическая схема по регистрации сверхбыстрого ОЭК [11-15,1719]. Лазерный импульс делился на пробный и накачивающий в соотношении $1: 9$. Угол между линейными поляризациями импульсов составил $45^{\circ}$. Далее лучи фокусировались линзой на кювету с исследуемой жидкостью, после чего с помощью диафрагмы выделялся пробный пучок. Состояние среды после воздействия импульса накачки регистрировалось по изменению интенсивности пробного импульса, который проходил через жидкость, расположенную между скрещенными поляризаторами. Таким образом, величина сигнала ОЭК была пропорциональна наведенной оптической анизотропии среды. При регистрации сверхбыстрого оптического эффекта Керра использовались известные методы оптического гетеродинирования и синхронного детектирования. Все эксперименты проводились при комнатной температуре.

Регистрация с применением синхронного детектирования осуществлялась без учета фазы. В этом случае наблюдаемый сигнал $S_{\mathrm{det}}(\tau)$ связан с сигналом сверх- быстрого ОЭК $S(\tau)$ соотношением

$$
S_{\mathrm{det}}(\tau)=\left|S(\tau)+C_{\mathrm{det}}\right|
$$

где аппаратная постоянная $C_{\operatorname{det}}$ диктуется условиями регистрации.

В качестве среды мы выбрали прозрачные в области $790 \mathrm{~nm}$ чистые, профильтрованные (размер пор фильтра $0.2 \mu \mathrm{m})$ жидкости толуола $\mathrm{C}_{7} \mathrm{H}_{8}$ и раствора комплекca $\mathrm{Eu}\left(\mathrm{CPDk}_{3-\mathrm{Ph}}\right)_{3} \mathrm{bpy}_{1-1}$ в толуоле с концентрацией $10^{-2} \mathrm{~mol} / \mathrm{l}$, которые помещались в кварцевой кювете толщиной $2 \mathrm{~mm}$.

\section{Результаты и дискуссия}

Сигналы ОЭК в чистом толуоле и растворе комплекса $\mathrm{Eu}\left(\mathrm{CPDk}_{3-\mathrm{Ph}}\right)_{3} \mathrm{bpy}_{1-1}$ в толуоле представлены на рис. 2 и 3 в субпикосекундном и пикосекундном диапазонах. Из рис. 2 видно, что сигналы обеих жидкостей идентичны в субпикосекундном диапазоне. Анализ оптических откликов, формирующих сигнал ОЭК в толуоле, в деталях представлен в литературе [20-23]. Таким образом, рис. 2 показывает, что редуцированная рамановская спектральная плотность, которая характеризует низкочастотные молекулярные либрации (вращательные колебания около равновесного положения) и внутримолекулярные колебания, одинакова для чистого толуола и раствора комплекса $\mathrm{Eu}\left(\mathrm{CPDk}_{3-\mathrm{Ph}}\right)_{3} \mathrm{bpy}_{1-1}$ в толуоле.

Напротив, пикосекундные составляющие сигналов ОЭК, показанные на рис. 3, значительно отличаются. Эти части сигнала ОЭК обусловлены наведенной ориентационной анизотропией жидкости, которая симулирует-

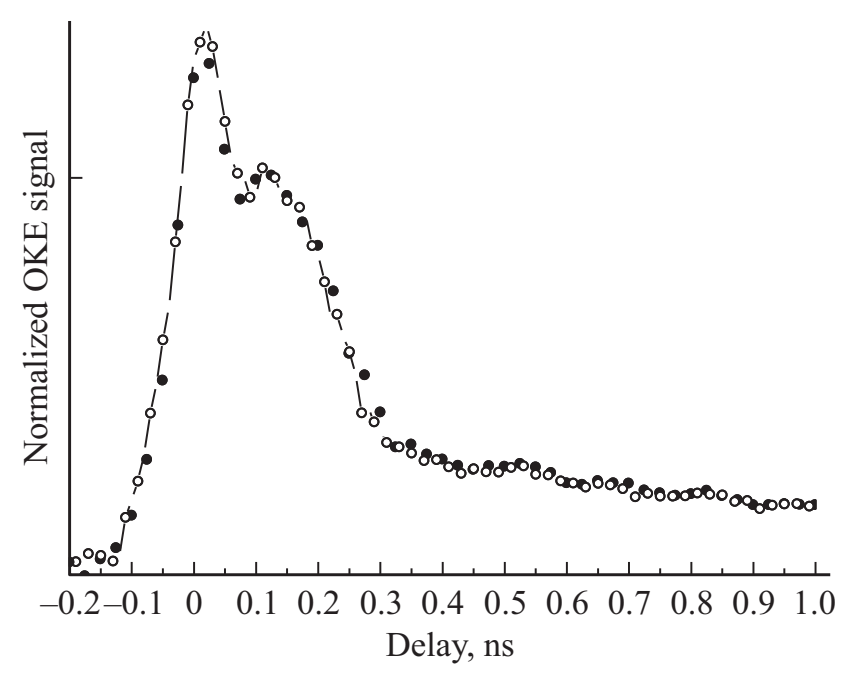

Рис. 2. Субпикосекундный диапазон сигнала сверхбыстрого ОЭК в чистом толуоле $\mathrm{C}_{7} \mathrm{H}_{8}$ (заполненные окружности) и растворе комплекса $\mathrm{Eu}\left(\mathrm{CPDk}_{3-\mathrm{Ph}}\right)_{3} \mathrm{bpy}_{1-1}$ в толуоле с концентрацией $10^{-2} \mathrm{~mol} / \mathrm{l}$ (пустые окружности) при возбуждении импульсами длительностью 45 fs. 


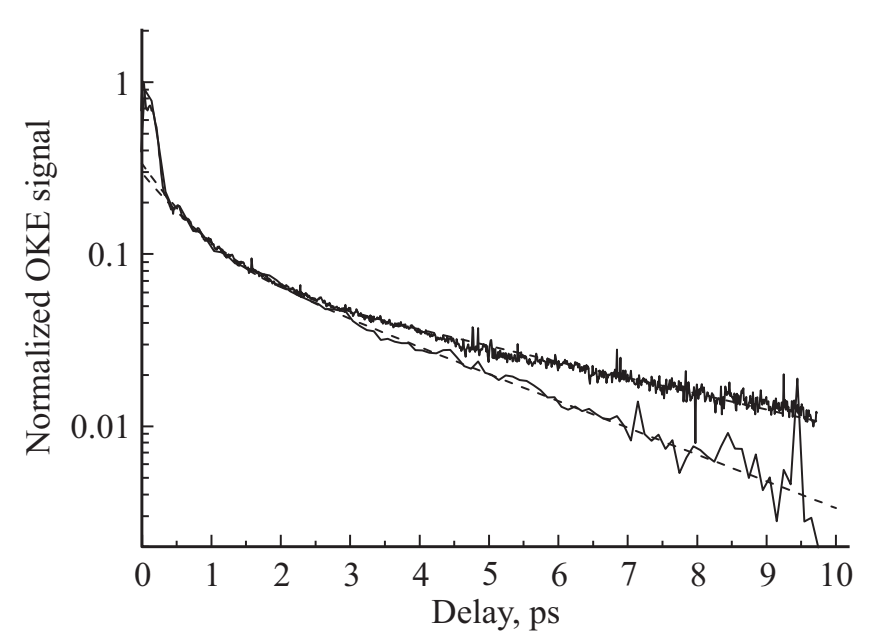

Рис. 3. Пикосекундный диапазон сигнала сверхбыстрого ОЭК в чистом толуоле $\mathrm{C}_{7} \mathrm{H}_{8}$ (верхняя кривая) и растворе комплекса $\mathrm{Eu}\left(\mathrm{CPDk}_{3-\mathrm{Ph}}\right)_{3} \mathrm{bpy}_{1-1}$ в толуоле (нижняя) с концентрацией $10^{-2} \mathrm{~mol} / 1$ при возбуждении импульсами длительностью $45 \mathrm{fs}$. Сплошная линия - экспериментальные данные, штриховая линия - ориентационный вклад, вычисленный согласно (3).

ся функцией ориентационного отклика [11-14]:

$$
\begin{aligned}
r_{\text {or }}(t) \sim & \left(A \exp \left(-\frac{t}{\tau_{\text {or }}^{(1)}}\right)\right. \\
& \left.+(1-A) \exp \left(-\frac{t}{\tau_{\text {or }}^{(2)}}\right)\right)\left(1-\exp \left(-\frac{t}{\gamma}\right)\right),
\end{aligned}
$$

где $\tau_{\mathrm{or}}^{(i)}$ - времена релаксации, обусловленные механизмами вращательной диффузии, константа $A$ определяет относительный вклад слагаемых в выражении (2), а параметр $\gamma$ симулирует нарастание ориентационного отклика, связанное с инерциальным характером молекулярных вращений. Сигнал ОЭК в пикосекудном диапазоне имеет вид

$$
S(\tau) \sim \int_{-\infty}^{\infty} r_{\mathrm{or}}(t) G(t-\tau) d t
$$

где $G(t)$ - автокорреляционная функция огибающей интенсивности лазерного импульса. Учитывая, что времена ориентационной релаксации $\tau_{\text {or }}^{(i)}$ на порядки превышают длительность лазерных импульсов (45 fs),

$$
\int_{-\infty}^{\infty} r_{\mathrm{or}}(t) G(t-\tau) d t \cong r_{\mathrm{or}}(\tau) \int_{-\infty}^{\infty} G(t-\tau) d t
$$

выражение (3) принимает вид

$$
\left.S(\tau) \sim\left(A \exp \left(-\frac{\tau}{\tau_{\text {оr }}^{(1)}}\right)\right)+(1-A) \exp \left(-\frac{\tau}{\tau_{\text {оr }}^{(2)}}\right)\right),
$$

где для простоты анализа в пикосекундном диапазоне принято, что нарастание ориентационного откли- ка происходит мгновенно: $\gamma \ll 1$ ps. Рисунок 3 показывает, что набор следующих параметров описывает ориентационный вклад сигналов ОЭК: $A_{\text {olt }}=0.25$, $\tau_{\mathrm{or}, \mathrm{tol}}^{(1)}=4.7 \mathrm{ps}$ и $\tau_{\mathrm{or}, \mathrm{tol}}^{(2)}=0.6 \mathrm{ps}$ для толуола; $A_{\mathrm{sol}}=0.4$, $\tau_{\mathrm{or}, \mathrm{sol}}^{(1)}=2.8 \mathrm{ps}$ и $\tau_{\mathrm{or}, \mathrm{sol}}^{(2)}=0.6 \mathrm{ps}$ для раствора комплекса $\mathrm{Eu}\left(\mathrm{CPDk}_{3-\mathrm{Ph}}\right)_{3} \mathrm{bpy}_{1-1}$ в толуоле.

Наличие в (4) двух экспоненциально затухающих слагаемых с временами $\tau_{\text {or }}^{(1)}$ и $\tau_{\text {or }}^{(2)}$ говорит о том, что в релаксации ориентационной анизотропии могут принимать участие два разных процесса вращательной диффузии. В случае толуола это может быть обусловлено сложной геометрией молекулы с разными моментами инерции относительно своих главных осей и локальным окружением, формирующим разные потенциальные барьеры относительно разных осей вращения. Наши эксперименты показывают, что комплексы $\mathrm{Eu}\left(\mathrm{CPDk}_{3-\mathrm{Ph}}\right)_{3} \mathrm{bpy}_{1-1}$ существенным образом влияют только на один из двух процессов вращательной диффузии: константа $\tau_{\text {or }}^{(1)}$ изменяется от значения 4.7 ps в чистом толуоле до значения $2.8 \mathrm{ps}$ в растворе комплекса $\mathrm{Eu}\left(\mathrm{CPDk}_{3-\mathrm{Ph}}\right)_{3} \mathrm{bpy}_{1-1}$ в толуоле; константа $\tau_{\text {or }}^{(2)}$ при этом не меняется.

Известно, что локальная структура жидкости обусловлена межмолекулярными взаимодействиями молекулы с ближайшим окружением, которые задают её равновесную ориентацию в потенциальной яме [24,25]. Вероятность $w$ перехода молекулы в новое ориентационное положение зависит от величины потенциального барьера $U$ :

$$
w \sim \exp (-U / k T),
$$

где $k-$ константа Больцмана, $T-$ температура. Время релаксации ориентационного отклика обратно пропорционально этой вероятности $\tau_{\text {or }}^{(1)}=g \exp (U / k T)$, где $g$ - предэкспоненциальный фактор. Идентичные части сигналов ОЭК в субпикосекундном диапазоне для обеих жидкостей (рис. 2) свидетельствуют, что спектр молекулярных либраций (связанный с редуцированной рамановской спектральной плотностью) не меняется при переходе от чистого толуола к раствору комплекса $\mathrm{Eu}\left(\mathrm{CPDk}_{3-\mathrm{Ph}}\right)_{3} \mathrm{bpy}_{1-1}$. Уместно предположить, что влияние комплексов $\mathrm{Eu}\left(\mathrm{CPDk}_{3-\mathrm{Ph}}\right)_{3} \mathrm{bpy}_{1-1}$ на локальную структуру растворителя сказывается главным образом на величине потенциального барьера, но не на форме потенциальной ямы. Предположение о том, что предэкспоненциальный фактор $g$ остается прежним, позволяет сделать грубую оценку изменения потенциального барьера $\Delta U$ :

$$
\Delta U \approx k T \ln \left(\frac{\tau_{\mathrm{or}, \mathrm{tol}}^{(1)}}{\tau_{\mathrm{or}, \mathrm{sol}}^{(1)}}\right),
$$

где значение $\Delta U$ составляет величину $0.5 k T$.

\section{Заключение}

Проведен сравнительный анализ вращательной динамки молекул толуола в чистой жидкости толуола и 
растворе комплексов $\mathrm{Eu}\left(\mathrm{CPDk}_{3-\mathrm{Ph}}\right)_{3} \mathrm{bpy}_{1-1}$ в толуоле на основе экспериментов по регистрации сверхбыстрого ОЭК в суб- и пикосекундном диапазонах. Показано, что сигнал ОЭК не является суммой двух независимых оптических вкладов, соответствующим ансамблям молекул толуола и комплексов $\mathrm{Eu}\left(\mathrm{CPDk}_{3-\mathrm{Ph}}\right)_{3} \mathrm{bpy}_{1-1}$. Более того, низкая концентрация комплексов $\mathrm{Eu}\left(\mathrm{CPDk}_{3-\mathrm{Ph}}\right)_{3} \mathrm{bpy}_{1-1}$ не позволяет регистрировать их вклад в сигнал ОЭК на фоне интенсивного вклада молекул толуола. Благодаря этому нам удалось проследить влияние комплексов $\mathrm{Eu}\left(\mathrm{CPDk}_{3-\mathrm{Ph}}\right)_{3} \mathrm{bpy}_{1-1}$ на локальную структуру раствора.

Сравнительный анализ показал, что взаимодействие комплексов $\mathrm{Eu}\left(\mathrm{CPDk}_{3-\mathrm{Ph}}\right)_{3} \mathrm{bpy}_{1-1}$ с молекулами толуола главным образом сказывается на одном из процессов вращательной диффузии: время ориентационной релаксации молекул толуола в растворе составляет величину $\tau_{\text {orsol }}^{(1)}=2.8 \mathrm{ps}$, а в чистом толуоле $\tau_{\text {or,tol }}^{(1)}=4.7$ ps. При этом второй процесс вращательной диффузии $\left(\tau_{\text {or,sol }}^{(2)} \cong \tau_{\text {or,tol }}^{(2)} \cong 0.6 \mathrm{ps}\right)$ и спектр молекулярных либраций остаются неизменными. Это позволяет предположить, что основное влияние комплексов на локальную структуру раствора состоит в уменьшении величины потенциального барьера в первом процессе вращательной диффузии (который, вероятно, следует ассоциировать с поворотами молекул толуола вокруг одной из главных осей вращения) на величину $0.5 k T$ без существенного изменения формы локальных потенциалов молекул толуола. Более детальное понимание механизмов изменения локальной структуры жидкости требует дополнительных экспериментальных и теоретических исследований.

\section{Список литературы}

[1] Brito H.F., Malta O.L., Felinto M.C.F.C., Teotonio E.E.S. // The Chemistry of Metal Enolates. / Ed. by Zabicky J. England: John Wiley \& Sons Ltd., 2009. V. 1. P. 131-184.

[2] Binnemans K. // Chem. Rev. 2009. V. 109. P. 4283-4374.

[3] Лапаев Д.В., Никифоров В.Г, Князев А.А. и др. // Опт. и спектр. 2008. Т. 104. № 6. С. 939 [Opt. Spectrosc. 2008. V. 104. P. 851].

[4] Лапаев Д.В., Никифоров В.Г., Сабиуллин Г.М. и др. // Журн. структур. химии. 2009. Т. 50. № 4. С. 809 [J. Struct. Chem. 2009. V. 50. P. 775].

[5] Lapaev D.V., Nikiforov V.G., Safiullin G.M. et al. // Opt. Mater. 2014. V. 37. P. 593.

[6] Lapaev D.V., Nikiforov V.G., Safiullin G.M. et al. // J. Lumin. 2016. V. 175. P. 106.

[7] Lapaev D.V., Nikiforov V.G., Safiullin G.M. et al. // J. Lumin. 2018. V. 194. P. 407.

[8] Lapaev D.V., Nikiforov V.G., Lobkov V.S. et al. // Opt. Mater. 2018. V. 75. P. 787.

[9] Лапаев Д.В., Никифоров В.Г, Лобков В.С. и др. // Изв. РАН. Сер. физ. 2018. Т. 82. № 8. С. 124.

[10] Hong Y., Lam J.W.Y, Tang B.Z. // Chem. Soc. Rev. 2011. V. 40. P. 5361.

[11] Kinoshita S., Kai Y., Ariyoshi T., Shimada Y. // Intern. J. Modern Physics B. 1996. V. 10. P. 1229.
[12] Shirota H., Kato T. // J. Phys. Chem. A. 2011. V. 115. P. 8797.

[13] Zhong Q., Fourkas J.T. // J. Phys. Chem. B. 2008. V. 112. P. 15529.

[14] Hunt N.T., Jaye A.A., Meech S.R. // Phys. Chem. Chem. Phys. 2007. V. 9. P. 2167.

[15] Самариев В.В., Никиборов В.Г. Фемтосекундная лазерная спектроскопия. М.: Тровант, 2017. С. 402.

[16] Knyazev A.A., Karyakin M.E., Romanova K.A. et al. // Eur. J. Inorg. Chem. 2017. P. 639.

[17] McMorrow D. // Opt. Comm. 1991. V. 86. P. 236.

[18] McMorrow D., Lotshaw W.T. // Chem. Phys. Lett. 1990. V. 174. P. 85.

[19] McMorrow D., Lotshaw W.T. // J. Phys. Chem. 1991. V. 95. P. 10395.

[20] Jaye A.A., Hunt N.T., Meech S.R. // Langmuir. 2005. V. 21. P. 1238.

[21] Hunt N.T., Jaye A.A., Meech S.R. // Phys. Chem. Chem. Phys. 2007. V. 9. P. 2167.

[22] Shmelev A.G. et al. // EPJ Web of Conferences. EDP Sciences, 2015. V. 103. P. 08003.

[23] Zharkov D.K. et al. // J. Phys.: Conference Series. IOP Publishing, 2016. V. 714. N 1. P. 012018.

[24] Френкель Я.И. Кинетическая теория жидкостей. М.: АН CCCP, 1945. C. 424.

[25] Burshtein A.I., Temkin S.I. Spectroscopy of Molecular Rotation in Gases and Liquids. Cambridge, New York, 1994. 